Ernährung in der Schule

\title{
Weg vom Zeigefinger
}

\section{OSSENA ist eines von sechs Projekten im Bereich von Agrarpolitik und Ernäh- rung, die im Rahmen der Sozialökologischen Forschung des Bundesministeriums für Bildung und Forschung durchgeführt werden. Hauptgegenstand von OSSENA ist die Erforschung der Bedingungen und Möglichkeiten für Veränderungen von Ernährungsverhalten und im Weiteren von Ernährungskultur.}

$\mathrm{D}$ Von Veronika Nölle as OSSENA-Projekt wird von den Lehrstïhlen Unternehmensführung sowie Marketing der Universität Oldenburg geleitet, weil gerade die Anbieterkulturen zum Thema gemacht werden sollen. Es besteht aus sechs Modulen: Landwirtschaft, Handel, Gastronomie, Großverbraucher, Aktionen im öffentlichen Raum, Schule und Pädagogik sowie einem Panel mit vierzig Haushalten.

Wenn Kinder alleine essen müssen, schmeckt es ihnen weniger und die Zeit der Nahrungsaufnahme wird drastisch verkürzt. Dabei äußern sich die Mädchen häufiger über ihre Gefühle beim Essen als die Jungen. Bei beiden Geschlechtern ist Fast-Food oft cool, aber auch das, was die Mama kocht. Allerdings setzt sich auch die Erkenntnis durch, dass Fast-Food ungesund ist, vor allem aber der Figur schadet, zaghafte Absetzbewegungen sind spürbar - Äpfel dagegen sind bei Mädchen der Renner. Und Kinder und Jugendliche sind begeisterte Köche, wenn man sie lässt.

\section{- Essen in der Familie}

In kaum einer Familie ist das Thema Essen nicht präsent. Betrachtet man, was als Angebot auf den häuslichen Tisch kommt, so ist es trotz aller Worte für Obst und Gemüse die traditionelle Hausmannskost und mit zunehmendem Alter der Kinder eine Anpassung an deren vermeintlichen Geschmack von Pommes, Pizza, Nudeln und Döner. Oft wird gesundes Essen auch gleichgesetzt mit BIO-Kost, und die ist nun besonders uncool. Hier werden, auch bei den beteiligten Erwachsenen, kulturelle Gräben deutlich. Das sind jedenfalls die ersten Erfahrungen aus dem Teilmodul Pädagogik des OSSENA-Projektes.

In vier Grundschul- und fünf Gesamtschulklassen in der Region Ostfriesland hat das Teilmodul Schule/ Pädagogik je zwei Projektwochen mit insgesamt 45 Unterrichtsstunden pro Klasse zum Thema Ernährungskultur in der Schule durchge- führt. Die Eltern der rekrutierten Schulen stellen die Träger des Haushaltepanels, über das die Einflussfaktoren ermittelt werden. Zudem liegen Supermärkte der Praxispartner und beteiligte landwirtschaftliche Direktvermarkter in unmittelbarer Nähe der Schulen und der Wohnorte.

Unsere Ausgangsfrage lautete: Welche Aspekte von Ernährungskultur lassen sich dauerhaft in den Schulalltag integrieren, und wie wirken sich die schulischen Interventionen auf den (Ernährungs-) Alltag in den Familien aus? Projektwochen lösen meistens Begeisterung aus, weil die Schülerinnen und Schüler vom Lernalltag befreit sind. Bei OSSENA wurde jedoch mit viel Spaß in einem ständigen Wechsel zwischen Theorie und Praxis hart gearbeitet.

Die vorhandenen Unterrichtsmaterialien zur Ernährungskultur und zu Ernährungsgewohnheiten wurden zunächst nach den Empfehlungen des europäischen Handbuchs ,Healthy eating for young people in Europe" ausgewählt und in eine Unterrichtskonzeption integriert, einige wurden neu entwickelt oder verändert. Die Einheiten wurden nach folgenden Überlegungen konzipiert und durchgefïhrt:

- Geruch, Geschmack und Atmosphäre prägen die Ernährungsgewohnheiten und das Essverhalten. Die Schüler/innen sollten erfahren, dass es einen Zusammenhang zwischen Ernährung und Emotionen gibt. Sie sollten mit (neuen) Geschmackserlebnissen konfrontiert werden und diese geschmacklich und gefühlsmäßig einordnen können. Wie ist die Beschaffenheit? „Es riecht, schmeckt, hört, fühlt sich an und erinnert mich....." Wann esse ich gerne und warum, was verdirbt mir den Appetit?

- Ess- und Ernährungsgewohnheiten haben sich verändert. Was hat sich historisch verändert in den Familien, bei den Schönheitsidealen und den Ernährungsgewohnheiten? Den Schüler/innen sollte bewusst werden, dass sich viele unterschiedliche Ernährungsstile mit den Verände- rungen des Konsumverhaltens entwickelt haben, dass heute nebeneinander existierende Ernährungskulturen Ausdruck einer individualisierten Gesellschaft sind und ihre eigenen Vorlieben nur einen Teil davon darstellen.

- Ernährung ist auch ein Mittel der Selbstinszenierung. Was ist in - was out? Ob Mode, Musik, Freizeitgestaltung, Körperkult oder Ernährung, sie sind Mittel von Selbstinszenierungen, durch die unter anderem Distinktionen oder Identitäten geschaffen werden.

- Die Wege unserer Nahrung wurden thematisiert. Was wird von wo, wohin transportiert, wie lässt sich der Verlust zum Beispiel von Vitaminen durch Lagerung und lange Transporte nachweisen? Wie hoch ist die Emissionsbelastung, was heißt ,fair gehandelt"? Die Schüler/innen haben über Supermarkterkundungen und Besuche auf Höfen einen Eindruck von Qualität, Sorten, es gibt etwa über 150 ostfriesische Apfelsorten, im Supermarkt aber nur fünf eingeflogene, und dem Arbeitsaufwand ihrer Herstellung bekommen.

- Und selbstverständlich wurde ständig gekocht, probiert, präsentiert und in einer Festtagsatmosphäre alles gemeinsam verzehrt. Bei ,was ist in - was ist out" tauchten plötzlich Pfannkuchen als besonders „in“ auf. Es geht also - die Schülerinnen und Schüler sind gut zu motivieren, wenn sie nicht belehrt, sondern moderierend begleitet werden.

Bis zum Sommer 2005 wird die Auswertung der einzelnen Unterrichtselemente vorliegen. Die Lehrkräfte haben in den unterschiedlichsten Fächern bereits Elemente der OSSENA-Einheiten eingebaut. Sie haben aber auch den dringenden Wunsch geäußert, die Einheiten fortzusetzen oder in den unteren Klassen zu wiederholen. Auf Seiten des Projektes gibt es Überlegungen, in der Region in Verbindung mit der wissenschaftlichen Forschung einen Wissenspool mit regionalen Institutionen aufzubauen, der den Schulen beratend zur Seite steht, und vielleicht Durchführungsangebote zu entwickeln, die allerdings finanzielle Unterstïtzung erfordern.

Die Autorin
Veronika Nölle ist wissenschaftliche Mitarbeiterin im
OSSENA-Projekt.
Kontakt: Dr. Dirk Fischer, Carl von Ossietzky Uni-
versität Oldenburg, Institut für BWL und Wirtschafts-
pädagogik, 26111 Oldenburg. Tel. 0441-7984457,
E-Mail: dirk.fischer@uni-oldenburg.de,
www.ossena-net.de


(c) 20I0 Authors; licensee IÖW and oekom verlag. This is an article distributed under the terms of the Creative Commons Attribution Non-Commercial No Derivates License (http://creativecommons.org/licenses/by-nc-nd/3.o/), which permits unrestricted use, distribution, and reproduction in any medium, provided the original work is properly cited. 